\title{
Biomimetic chemistry: radical reactions in vesicle suspensions
}

\author{
Chryssostomos Chatgilialoglu and Carla Ferreri \\ ISOF, Consiglio Nazionale delle Ricerche, Bologna \\ Italy
}

\section{Introduction}

Chemical reactivity represents the fundamental basis for studying processes in life sciences. In particular, the last years have seen the affirmation of the interdisciplinary field of chemical biology, which has motivated a strong interest in modeling chemical reactivity of biological systems, that is, improving chemical methodologies and knowledge in order to understand complex reaction pathways related to cellular processes. In this context the reactivity of free radicals revealed its enormous importance for several biological events, including aging and inflammation (Cutler \& Rodriguez, 2003), therefore the modeling of free radical reactions under naturally occurring conditions has become a basic step in the research of fundamental mechanisms in biology. The assessment of modes of free radical reactivity has been found to be important at least in three areas: i) the examination of interactions at a molecular level leading to the discovery of radical-based processes involved in enzymatic activities, e.g., ribonucleotide reductase (Reichard \& Ehrenberg, 1983), cyclooxygenase (Marnett, 2000), the drug effects of antitumorals (Goldberg, 1987), vitamin activities (Buettner, 1993); ii) The clarification of free radical processes that can lead to damage of biomolecules, together with the individuation of products, opening the way for the evaluation of the in vivo damage and its role in the overall cellular status (Kadiiskaa et al., 2005; Pryor \& Godber, 1991); iii) the knowledge of free radical mechanisms allowing for new strategies to be envisaged in order to control the level of the damage and fight against the negative consequences (Halliwell \& Gutteridge, 2000). These three main areas represent the core studies of free radicals using biomimetic models.

In the last decade our group has developed the subjects of lipid and protein damages under biomimetic conditions, and in particular envisaged novel damage pathways for the transformation of these important classes of biomolecules. In this chapter biomimetic models will be examined, also mentioning work previously done by others in the field and the advancements carried by us. Information will be given on liposome vesicles, which is the basic context for examining free radical reactivity in heterogenous conditions, where the partition of the reactants occurs between the lipid and the aqueous environments, and this can influence the biological effects. The regioselectivity driven by the supramolecular organization of lipids in the vesicle double layer is another feature of the biomimetic model that has been related to the formation of trans lipids, specific markers of radical stress in cell 
membranes. Moreover, biomimetic chemistry has been developed on small radical species able to enter the hydrophobic compartment of the vesicle, evidencing the concomitant event of desulfurization involving sulfur-containing amino acid residues. Finally, in this chapter the biomimetic models will be highlighted also as a very useful tool where possible scenarios of biological consequences can be foreseen, such as those deriving from the study of the minimal cell to develop a biological life.

\section{Modeling radical reactions in vesicles}

The model treated in this chapter is a lipid vesicle, which is used as model of the cell membrane. The natural structure of cell membranes is a double layer of phospholipids, which are amphiphilic molecules of general formula shown in Figure 1, capable of selforganization. The hydrophobic part mostly consists of fatty acid residues, that are carboxylic acids with a long hydrocarbon chain (up to 26 carbon atoms), saturated or unsaturated with up to six double bonds. A specific structural feature of naturally occurring mono- and polyunsaturated fatty acid (MUFA and PUFA) residues is the cis double bond geometry, whereas PUFA have the characteristic methylene-interrupted motif of unsaturated chain. Examples of mono- and polyunsaturated fatty acid (MUFA and PUFA) structures and also of some trans isomers are shown in Figure 2, with the common names and the abbreviations describing the position and geometry of the double bonds (e.g., 9cis or 9trans), as well as the notation of the carbon chain length and total number of unsaturations (e.g., C18:1) (Vance \& Vance, 2002). It is worth noting that being the cis geometry connected with biological activities, this feature is strictly controlled during MUFA and PUFA biosynthesis by the regiospecific and stereoselective enzymatic activity of desaturases (Fox et al, 2004).

In the free radical reactivity the double bonds and bis-allylic positions are the moieites that undergo the chemical transformations, and these processes have been ascertained to play relevant roles in pathological processes and aging. The subject of lipids and free radicals is typically interdisciplinary because it involves all disciplines of life sciences. In this respect, it was looked for appropriate models of free radical reactivity in membranes, and liposomes are the universally accepted models for cell membranes as they can closely simulate the bilayer structure. Liposomes can be represented as shown in Figure 3, i.e., a double layer formed by spontaneous organization of the phospholipid components in water, delimiting an aqueous cavity. The fatty acid tails can be saturated or unsaturated, and the disposition of the double bonds in the vesicle depends on the supramolecular arrangement of the bilayer. Multilayer vesicles (MLV), having an onion-like structure, are obtained from dry lipids added with an aqueous medium and vortexed (New, 1990; Lasic, 1993). However, this type of vesicle are not the best membrane models, since the observation of the diffusion phenomenon through several layers cannot be directly extrapolated to the passage across a single bilayer, like it occurs in natural membranes. Monolamellar vesicles are the closest model to membranes, and they can be formed by different techniques, such as the extrusion (MacDonald et al., 1991) and the injection methodologies (Domazou \& Luisi, 2002). 


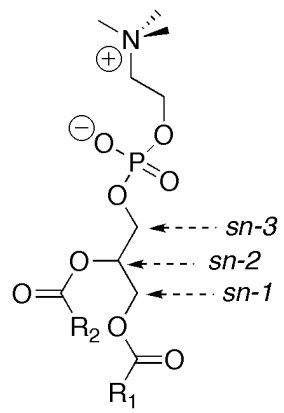

Fig. 1. The general structure of L- $\alpha$-phosphatidylcholine (PC), with two hydrophobic fatty acid chains in the positions sn- 1 and sn-2 of L-glycerol and the phosphorous-containing polar head-group in sn-3 position.<smiles>CCCCCCCCCCCCCCCC(=O)O</smiles><smiles>CCCCCCCC/C=C\CCCCCCCC(=O)O</smiles><smiles>CCCCCCCCC=CCCCCCCCC(=O)O</smiles><smiles>CCCCC/C=C\C/C=C\CCCCCCCC(=O)O</smiles><smiles>CCCCC/C=C\C/C=C/CCCCCCCC(=O)O</smiles><smiles>CCCCCC=CCC#CCC=CCC=CCCCC(=O)O</smiles>
arachidonic acid or 5 cis, 8 cis, 11 cis, 14 cis-C20:4<smiles>CCCCCC=CCC#CCC=CCC=CCCCC(=O)O</smiles>

5 trans, 8 cis, 11 cis, 14 cis-C20:4

Fig. 2. Some of the most common mono- and polyunsaturated fatty acid (MUFA and PUFA) structures, with their common names and the abbreviations describing the position and geometry of the double bonds (e.g., 9cis), as well as the notation of the carbon chain length and total number of unsaturations (e.g., C18:1). 

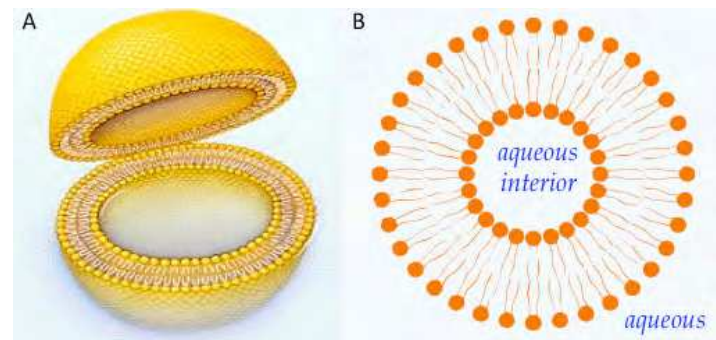

Fig. 3. Large unilamellar vesicles (LUV)

Among the lipid molecules used for liposome experiments, glycerophospholipids are relevant that account for approximately $60 \mathrm{~mol} \%$ of total lipids in the organism, and are made of the glycerol backbone having a polar head and two hydrophobic fatty acid residues (see Figure 1). Synthetic phospholipids can have both fatty acid chains as monounsaturated residues (for example, dioleoylphosphatidylcholine DOPC with two residues of oleic acid, 9cis-C18:1), or alternatively, one unsaturated and the other saturated fatty acid chains (for example, 1palmitoyl-2-oleoylphosphatidylcholine POPC, with one chain of saturated fatty acid residues of palmitic acid 16:0, and the other chain of the monounsaturated cis fatty acid, oleic acid 9cis18:1), the saturated one not participating to the free radical transformation, but having the role of internal standard for the quantitative analysis of the reaction outcome. Phosphatidylcholines of natural origins can be also used, such as soybean or egg lecithins, that contain the fatty acid chains as mixtures of saturated, monounsaturated and polyunsaturated residues. For example, in egg lecithin the mean fatty acid composition is: palmitic acid (C16:0) $32 \%$, stearic acid (C18:0) 14.1\%, oleic acid (9cis-C18:1), vaccenic acid (11cis-C18:1) 1.2\%, linoleic acid (9cis,12cis-C18:2) 20\%, arachidonic acid (5cis,8cis,11cis,14cis-C20:4) 4.8\%. Lecithins can simulate much closer the various types of fatty acids present in the natural membranes. In all these compounds another difference with the natural structures consists of the polar head, which is generally chosen as choline, whereas mixtures of choline, serine, ethanolamine and sugar derivatives are present in the real membranes. Vesicle models present in the literature are made of multilamellar vesicles, obtained by a dry film of phospholipids simply added with water and vortexed to obtain a milky suspension. Sonication can provide for a rearrangement of the starting multilamellar organization into smaller vesicles, which can be considered small liposomes, quite monolamellar in the arrangement or nearly so. As previously noted, the multilayer organization of lipids can present differences, because the diffusion of species becomes a complex process through several layers. However, information of the physical properties of all these suspensions is available and one can choose the appropriate model, which offers the heterogeneous aqueous environment where oxidative processes can be examined under a complexity still similar to the biological medium.

Free radical reactivity studied with these biomimetic models has the advantage to use a scenario closely related to a biological environment, but still simplified and controllable. During the eighties the vesicle system started to be developed in different directions: for examining membrane dynamics and transitions, (Siminovitch et al., 1987; Wolff \& Entressangle, 1994) for the incorporation of proteins and the protein-lipid interactions or functioning (Gregoriadis, 1992), for studying delivery systems (Fendler \& Romero, 1977) and many other applications. In free radical research, vesicles were used essentially in two directions: i) the study of free radical-based processes involving directly the lipid 
components, mainly lipid peroxidation; ii) the effect of antioxidants or radical trapping agents toward radical damages to biomolecules. These aspects will be treated in the next sections. It must be underlined that experiments were also carried out with micelles and other aggregation forms involving lipid compounds, but the present chapter deals with the model closest to the membrane structure, therefore only vesicles formed by phospholipid bilayer are considered. It is also worth noting that the methodology of phospholipid vesicles has taken a while to be assessed and appropriately tuned to the experimental needs; for example, the characteristic of lipid monolamellarity is needed for simulating cell membranes, but the former models were multilamellar vesicles, and after more than two decades the results can be updated by more recent knowledge.

\subsection{Oxidative transformations of lipid vesicles and the antioxidant activity}

The fact that oxidative processes were found to be deeply involved in cell metabolism and also in its degradation pathways was stimulating research of the basic chemical mechanisms. Oxidation of polyunsaturated fatty acids (PUFA) by free radicals immediately acquired importance also as in vivo process, in particular membrane lipid damage caused either by radiation (Marathe \& Mishra, 2002; Mishra, 2004) or by chemical poisons $\left(\mathrm{CCl}_{4}\right.$, ethanol) (Kadiiskaa et al, 2005). Lipid polyunsaturated components are highly oxidizable materials, and membrane models have to be used to assess the phenomenon since PUFA are present also in all biological membranes and lipoproteins. In PUFA the most sensitive site to oxidative attack is the bis-allylic position, the methylene group located between two double bonds. Detailed studies of the products and mechanism of peroxidation started in the 70's by several research groups (Porter et al, 1979; Porter et al, 1980; Milne \& Porter, 2001). The first products to be individuated were the hydroperoxides derived from the corresponding peroxyl radicals (Figure 4). The mechanism of lipid peroxidation (a radical chain reaction) starts with the abstraction of hydrogen atom producing the bisallylic (or pentadienyl) radical $\mathrm{L}^{\bullet}$ (Figure 4 ). The reaction of $\mathrm{L}^{\bullet}$ with oxygen is close to a diffusion-controlled process, but is also reversible. Indeed, the peroxyl radical can undergo a very rapid fragmentation. Peroxyl radicals LOO' can abstract a hydrogen atom to produce lipid hydroperoxide (LOOH) together with "fresh" $\mathrm{L}^{\bullet}$ radicals to continue the chain. Termination steps occur either by radical-radical combination or by attacking other molecules, such as an antioxidant ( $\alpha$-tocopherol ) or proteins.

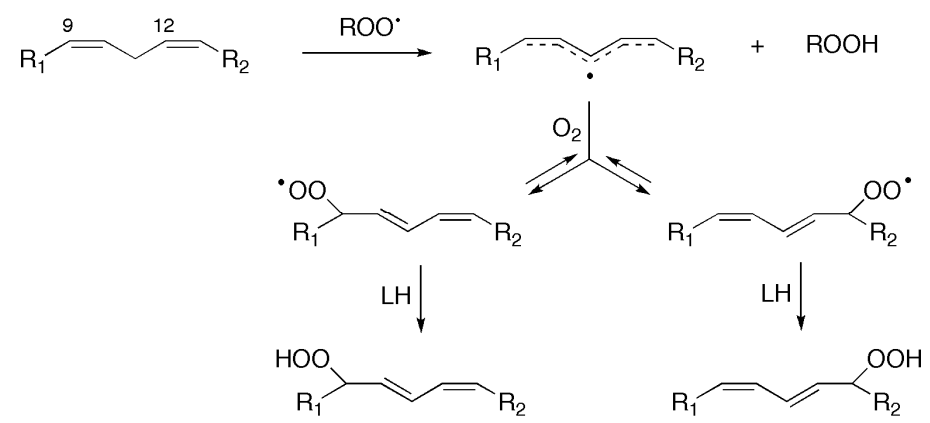

Fig. 4. Outline of the mechanism of lipid peroxidation with formation of kinetic-controlled trans-cis products 
The products of lipid peroxidation are not only hydroperoxides, but also conjugated dienes (Porter et al, 1979). Further decomposition of these products by the action of transition metals in their low oxidation state (i.e., $\mathrm{Fe}^{+2}$ ) leads to aldehydes and hydrocarbon endproducts, together with the subsequent combination of aldehydes to form adducts, all products that are used nowadays for testing and measuring the occurrence of oxidative stress in biological specimens (Esterbauer et al., 1989). By UV spectroscopy the quantification of conjugated dienes at 233 and $215 \mathrm{~nm}$ is used to follow accurately the initial stages of the process (Mihaljević et al., 1996).

The biomimetic models have been extremely useful to quantitate these events. The methodology includes the steps of preparation of vesicle suspensions and choice of the free radical initiation.

In heterogeneous systems the ability of PUFA to undergo chain oxidation (autoxidation) (Barclay et al., 1985) was examined in order to see whether differences can be found with the homogeneous solution. With these models different kinds of free radical conditions can be used, since an important point in the preparation of the experiments is the source of radical initiation. In case of the use of gamma or X-irradiations the initiation occurs in the aqueous compartment with formation of primary radical species from the interaction with water that can be quantified on the basis of the radiation dose. For example, the initiation by gamma irradiation of aqueous suspensions occurs by the Equation (1), where in parenthesis the radiation chemical yields in units of $\mu \mathrm{mol} \mathrm{J}^{-1}$ are shown.

$$
\mathrm{H}_{2} \mathrm{O} \stackrel{\gamma}{\longrightarrow} \mathrm{e}_{\mathrm{aq}^{-}}(0.27), \mathrm{HO} \cdot(0.28), \mathrm{H}^{\bullet}(0.06), \mathrm{H}^{+}(0.27), \mathrm{H}_{2} \mathrm{O}_{2}(0.07)
$$

The kinetics of reaction of ${ }^{\cdot} \mathrm{OH}$ and $\mathrm{e}_{\mathrm{aq}}{ }^{-}$with lecithin bilayers have been measured (Barber \& Thomas,1978). The rate for ${ }^{\bullet} \mathrm{OH}$ with lecithin is $5.1 \times 10^{8} \mathrm{M}^{-1} \mathrm{~s}^{-1}$, while $\mathrm{e}_{\mathrm{aq}}$ - rate is very slow. These rates are lower than those observed for similar reactions in homogeneous systems. This is explained in terms of the protective effect of the bilayer, this being especially true for $\mathrm{e}_{\mathrm{aq}}{ }^{-}$which does not readily leave the aqueous phase, and in terms of the restricted diffusion imposed on the reactive species by the bilayer. Long-term alteration in the model membrane following $\cdot \mathrm{OH}$ attack is manifested in terms of damage to the head group, increasing water penetration of the bilayer, and of cross-linking with the membrane, thereby restricting motion in the interior of the bilayer. Increased rigidity and "leakiness" of membranes is an expected consequence of radiation damage. In general, these processes modify the physical properties of the membranes, including the permeability to different solutes and the packing of lipids and proteins in the membranes, which in turn, influence membrane functions (Marathe \& Mishra, 2002; Schnitzer et al., 2007). A word of caution must be spent for the compounds used for measuring the vesicle properties, which have to be added at the end of the experiments. In fact, for example the fluorescent probe pyrene solubilized in the bilayer can react with $\cdot \mathrm{OH}$ and $\mathrm{e}_{\mathrm{aq}}^{-}\left(1.7 \times 10^{9} \mathrm{M}^{-1} \mathrm{~s}^{-1}\right.$ and $7 \times 10^{7} \mathrm{M}^{-1} \mathrm{~s}^{-1}$, respectively). Former experiments were reported with small liposomes obtained by sonication of a vesicle suspension made of natural phospholipids, extracted from mice liver cells. X-ray at two different doses ( 0.8 and $8 \mathrm{~Gy} / \mathrm{min}$,) in the presence and absence of oxygen, was used for a total $100 \mathrm{~Gy}$. Conjugated dienes and the main fatty acid residues were evaluated. The former were evaluated spectroscopically, as previously indicated, whereas the fatty acid composition was determined by workup of the liposome, extraction of lipids, 
transesterification to fatty acid methyl esters and gas chromatographic (GC) analysis (Konings et al., 1979). Under anoxic condition there is no dose effect, whereas the irradiation in the presence of oxygen (air bubbling) lead to extensive consumption, especially of the arachidonic and docosahexaenoic acid residues. In the same paper it was also advanced the protective effect of glutathione, cysteamine and $\alpha$-tocopherol, showing that the latter was the most effective. The radiation effect and lipid peroxidation were also assayed with gamma irradiation of soybean lecithin liposomes, and related to the dose-dependent formation of malondialdehyde (MDA) (Nakazawa \& Nagatsuka,1980). In the same paper the authors reported the resulting permeability of liposomes that is increasing linearly with the dose for the glucose efflux.

The kinetics of peroxidation can also be studied by free radical processes induced by an "external" generator of free radicals, like azo-compounds of general formula R-N=N-R, which decompose at a given temperature leading to radical $R^{\bullet}$ and $\mathrm{N}_{2}$. The azo-initiators are successfully used for radical processes in homogeneous systems, but in vesicle suspensions this methodology can result in some difficulties. In fact, the nature of the initiator can be hydrophilic or hydrophobic, and therefore the effect is governed by the diffusion of the species, i.e., by the balance between the effects of membrane properties on the rate constants of propagation and termination of the free radical peroxidation in the relevant membrane domains, represented by those domains in which the oxidizable lipids reside. Both these rate constants depend similarly on the packing of lipids in the bilayer, but influence the overall rate in opposite directions. This can be the reason for quite contrasting results reported in the literature. For example, linoleic acid, taken as typical example of unsaturated fatty acid, has a similar oxidizability in different media as determined by different procedures $\left(0.02-0.04 \mathrm{M}^{-1 / 2} \mathrm{~s}^{-1 / 2}\right)$ (Barclay, 1993). The systematic determination of oxidizability in the extended homologous series of PUFA and comparison with the literature values have been done, indicating an increase value by increasing the number of bisallylic carbons. The relationship in the series linoleic acid/linolenic acids/arachidonic acid/docosapentaenoic acid/ docosahexaenoic acid has been shown to be $1: \sqrt{2}: 2: 2 \sqrt{2}: 4$. On the other hand, for the autoxidation of egg lecithin using AIBN [azobis(isobutyronitrile)] as lipophilic radical initiator (Barclay \& Ingold, 1981) it is reported that the oxidizability of egg lecithin at $30{ }^{\circ} \mathrm{C}$ in vesicles is only $2.7 \%$ of that for the homogeneous material. It must be pointed out that the system used in those experiments was a lipid emulsion, with multilamellar vesicles, that could have influenced the viscosity of the medium and enhanced the self-termination of the initiator in the lipid bilayer, thus determining less efficiency of the peroxidation process.

The vesicle system and peroxidation process offered a good scenario also for examining the antioxidant activity. Indeed, the presence of an antioxidant network of enzymes and molecules that protects from free radical damages has been clearly demonstrated, and the consumption of these antioxidant defences has been linked to many pathological events (Halliwell \& Gutteridge, 2000). Again, in the liposome models the antioxidant properties and efficiency can be studied, in order to envisage their mode of action and, more importantly, the synergies that the molecular combination of different chemical mechanisms can provide, similarly to what occurs in the biological medium. Investigations focused first on natural compounds, and peroxidation processes were found to be successfully controlled by the activity of several molecules. Among them, vitamins and thiols give a quite complete scenario of the molecular properties required for an antioxidant. Natural vitamins constitute 
themselves a synergic network for the control of free radical processes; in the liposome models the combined effect of mode of action, partition coefficient and relative reactivity can be evaluated, which is different for each compounds. Vitamin E is one of the former compound to be studied and its mode of action is a chain-breaking process, due to the $\mathrm{H}$ atom donation from the phenolic hydroxyl group. By this way it can scavenge the peroxyl radicals stopping the chain propagation and the extensive decomposition of lipids. The partition of this compound is expected to occur in the lipophilic compartment, although a hydrophilic character can be present at the level of the hydroxyl group. Therefore, the location of this vitamin can be at the interface between the aqueous and the lipid compartments. In the liposome model this partition must be taken into account, since it is important to test both initiations, i.e., with lipid- and water-soluble azocompounds, AIBN and AAPH [(azobis(2-amidinopropane) dihydrochloride], respectively (Niki et al.,1985). Soybean multilamellar liposomes were oxidized in a similar manner with both initiation compounds, evaluated with the oxygen consumption methodology. When vitamin $\mathrm{E}$ or $\mathrm{C}$ was added in the AAPH-initiated oxidation the process was markedly suppressed. When vitamins are together added to the suspension, the first consumed is vitamin C, linearly with the time, followed by vitamin $E$ that starts to diminish when vitamin $C$ is consumed. In the AMVN-initiated process, vitamin $\mathrm{E}$ was clearly efficient in stopping the process, whereas vitamin $C$ did not affect the reaction course. Interestingly, the use of the two vitamins together were shown to ameliorate the induction period also in the AMVN experiment, thus indicating that, although vitamin $C$ cannot influence the formation of the lipid radicals within the bilayer, it can synergize with vitamin $\mathrm{E}$ activity prolonging its effect. These experiments were the first showing what is well known nowadays: vitamin $E$ is recycled by vitamin $C$. As far as the synergism is concerned, liposomes allowed for the study of other compounds, such as quinone compounds (coenzyme Q) and conjugated dienes (vitamin A, carotenoids, etc), to be used in combination for antioxidant strategies.

\subsection{Lipid isomerization and the vesicle effect on regioselectivity}

Figure 5 shows the reaction mechanism of free radical double bond isomerization that consists of a reversible addition of radical RS • to the double bond. Indeed, the reconstitution of the double bond is obtained by $\beta$-elimination of RS ${ }^{*}$ and the result is in favor of trans geometry, the most thermodynamically favorable disposition. The energy difference between the two geometrical isomers of prototype 2-butene is $1.0 \mathrm{kcal} / \mathrm{mol}$. It is worth noting that (i) the radical RS• acts as a catalyst for cis-trans isomerization, and (ii) positional isomers cannot be formed as reaction products because the mechanism does not allow a double bond shift (Chatgilialoglu \& Ferreri, 2005; Ferreri \& Chatgilialoglu, 2005). The effectiveness of cis-trans isomerization in the presence of the most common antioxidants has also been addressed. The high efficiency of all-trans retinol and ascorbic acid as antiisomerising agents in the lipophilic and hydrophilic compartments, respectively, parallels the well-assessed high reactivity of RS radicals towards these two antioxidants (Chatgilialoglu et al., 2002). Considering polyunsaturated substrates, the isomerization mechanism occurs as a step-by-step process depicted in Figure 6 for linoleate moiety, i.e., each isolated double bond behaves independently as discussed above (Ferreri et al., 2001). 


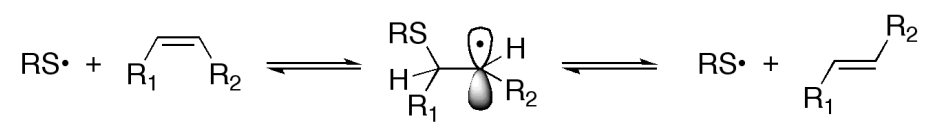

Fig. 5. The thiyl radical RS - acts as a catalyst for cis-trans isomerization by addition/elimination steps

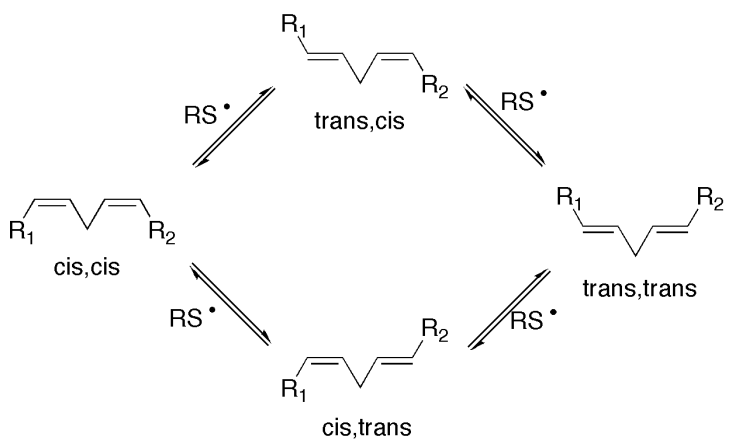

Fig. 6. Stepwise mechanism for the cis - trans isomerization of linoleate residues

Other types of free radicals (e.g., $\mathrm{RSe}^{\bullet}, \mathrm{RSO}_{2}{ }^{\circ}, \mathrm{NO}_{2}{ }^{\circ}, \mathrm{R}_{3} \mathrm{Sn}^{\bullet},\left(\mathrm{Me}_{3} \mathrm{Si}\right)_{3} \mathrm{Si}^{\bullet}$, etc.) and atoms (e.g., $\mathrm{Br}^{*}, \mathrm{I}^{\bullet}$, etc.) are known to induce cis-trans isomerization of double bonds by additionelimination steps (Jang et al., 1999; Chatgilialoglu \& Ferreri, 2005). However, the efficiency of the isomerization process strongly depends on the characteristics of the attacking radicals, and although another biologically important radical is $\mathrm{NO}_{2}{ }^{\circ}$, it should be added that thiols are known as the dominant 'sink' for $\mathrm{NO}_{2}{ }^{-}$in cell/tissues (Equation 2). The rate constant is close to $2 \times 10^{7} \mathrm{M}^{-1} \mathrm{~s}^{-1}$ with generation of thiyl radicals, therefore in the biological environment thiyl radicals are likely to be the most relevant isomerizing species (Chatgilialoglu et al., 2006).

$$
\mathrm{NO}_{2}^{\cdot}+\mathrm{RSH} \rightarrow \mathrm{NO}_{2}^{-}+\mathrm{RS} \cdot+\mathrm{H}^{+}
$$

It is worth noting at this point that a few years ago, the importance of trans fatty acids was known only in nutrition studies. In fact, the transformation of double bonds from the natural cis geometry to a variety of positional and geometrical trans isomers results from the processes of partial hydrogenation and deodorization used in food industry to obtain margarines and other fat shortenings. There are several books and reviews on this subject (Sébédio \& Christie, 1998), therefore here it is highlighted only that the free radical-mediated isomerization is an endogenous process which has nothing to do with the chemical manipulation of fats as origin of the trans lipid geometry.

The first report highlighting the lipid isomerization mechanism as a biologically meaningful process was from our group in 1999 (Ferreri et al., 1999). Using biologically relevant compounds and phospholipids, the occurrence of such a transformation was modeled under biomimetic conditions. The subject was of interest to other research groups and all work done in this area showed that thiyl radicals are efficient and effective isomerizing agents (Chatgilialoglu \& Ferreri, 2005; Ferreri \& Chatgilialoglu, 2005). In another review the subject 
of the thiyl radical production in biosystems and effects on lipid metabolism is summarized (Ferreri et al. 2005b).

Taking inspiration from the lipid peroxidation process extensively studied in liposomes, unsaturated lipid vesicles were envisaged as a good biomimetic model for the double-bond isomerization. Indeed, early reports on the use of glutathione, or other thiol compounds such as cysteine, as effective protective agents against the radiation-induced lipid peroxidation, did not mention the stability of the double-bond geometry (Konings et al., 1979; Prager et al., 1993). In our experiments large unilamellar vesicles obtained by extrusion technique (LUVET) with polycarbonate filter of $100 \mathrm{~nm}$ diameter were used, that form an almost transparent suspension, which is also suitable for studies under photolytic conditions. As pointed out also before, the aqueous and lipid phases are the two distinct compartments of this non-homogeneous system. There are several features to be taken into account for examining the reactivity of this system towards free radicals: i) the characteristic supramolecular arrangement of the lipid assembly, with the fatty acid chains of phospholipid molecules that form the hydrophobic core of the model membrane, and the polar heads that face the aqueous internal and external phases (see Figure 3); ii) the partition coefficient of compounds added to the system, which influences the distribution of the reactive species in the two compartments; iii) in particular, the location of the initiation step, that is, where the formation of an initial radical species, able to abstract the H-atom from the thiol group, occurs. As far as the lipid organization is concerned, there is a precise arrangement of the hydrophobic core, which can influence the position of the double bonds in the layer and the reactivity of the different fatty acids to the radical attack. This was found to be the case in the double bond isomerization, studied with an amphiphilic thiol, 2mercaptoethanol, that is, a compound able to diffuse without restriction from the aqueous phase to the lipid bilayer, and vice versa. A regioselective process resulted where the double bonds are not involved at the same extent by the radical isomerization. In particular, using vesicles made of egg yolk lecithin, it was possible to demonstrate that the double bonds located closest to the membrane polar region are the most reactive towards the attack of diffusing thiyl radicals (Ferreri et al., 2002; Ferreri et al., 2004a). In the case of linoleic acid residues in vesicles, the double bond in position 9 resulted more reactive than that in position 12. Also arachidonic acid residues in vesicles were more reactive than oleic and linoleic acids, and two positions, i.e., the double bonds in 5 and 8 over the four present in this compound, were transformed preferentially. The scenario could be different for other long-chain PUFA, depending on their supramolecular arrangement, and in this context isomerization by diffusible thiyl radical can act as a reporter, indirectly informing on the double bond disposition in the bilayer.

From the studies carried out so far, arachidonic acid residues in membrane phospholipids emerge as very important markers to be investigated, in order to distinguish endogenous trans isomers, formed by radical processes, from the exogenous trans isomers, derived from dietary contribution. In particular, investigation can be focused on erythrocyte membrane phospholipids, which are the preferential storage for arachidonic acid after biosynthesis. As matter of fact, the case of arachidonic acid is a seminal example of how it is possible to distinguish the endogenous isomerisation from the trans isomers contained in foods. Considering the biosynthetic paths of omega- 6 fatty acids represented in Figure 7, two double bonds (positions 11 and 14) originate from linoleic acid, the essential fatty acid precursor taken from the diet, whereas the two other double bonds (positions 5 and 8) are 
formed by desaturase enzymes, which produce selectively the cis unsaturation. It is evident that the double bonds 5 and 8 of arachidonic acid, can only have a cis configuration, unless in the membranes these positions are involved in an isomerization process by diffusible thiyl radicals and transformed into trans isomers.

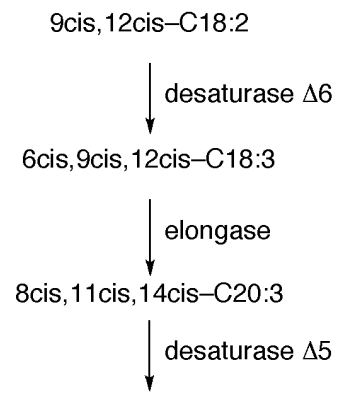

5 cis, 8 cis, 11 cis, 14 cis-C20:4

Fig. 7. Enzymatic fatty acid transformations of the omega 6 fatty acid pathway

A careful identification of membrane lipids containing arachidonic residues can be important for functional lipidomics, in order to achieve a clear understanding of the contribution from endogenous or exogenous processes. We extended the biomimetic investigation to biological systems, in order to prove the "endogenous" trans lipid formation under strictly physiological conditions. It is important to deal with "trans-free" conditions, which means that the presence of any external source of trans fatty acid isomers is carefully checked. Cell membrane lipid composition of human leukemia cell lines (THP-1) was monitored during incubation in the absence and presence of thiol compounds, ensuring that no contribution of trans compounds could come from the medium (Ferreri et al., 2004b). The experiments were based on the hypothesis that the normal cell metabolism includes several radical-based processes. Therefore, the intracellular level of sulfur-containing compounds could have produced a certain amount of thiyl radicals and consequently, caused a lipid isomerization. In parallel experiments, some thiol compounds were added in $\mathrm{mM}$ levels to the cell cultures during incubation, and the comparison of isomeric trends was done. Indeed, a basic content of trans lipids in THP-1 cell membranes was found during their growth before thiol addition, and by addition of the amphiphilic 2-mercaptoethanol, it was increased up to $5.6 \%$ of the main fatty acid residues. Even greater trans lipid formation was obtained by a radical stress artificially produced in the cell cultures added with thiol, and for example, by $\gamma$-irradiation a $15.5 \%$ trans content in membrane phospholipids was reached. The fatty acid residues most involved in this transformation were arachidonate moieties, and this result confirmed that these are the most important residues to be monitored in cells. The trans arachidonate content determined in THP-1 membrane phospholipids provides the first indication of the occurrence of an endogenous isomerization process, not confused with a dietary contribution, as previously explained. This opened new perspectives for the role of trans lipids in the lipidome of eukaryotic cells and was followed by several other investigations in living systems (Zambonin et al., 2006; Ferreri et al, 2005a; Puca et al., 2008). Actually, the formation of trans lipids can be evaluated in terms of relevant percentages within membranes, which means that not only can they 
have a negative effect for the lipid assembly, but also act as a signaling for triggering some protective responses in cells.

\section{Free radical-based tandem protein-lipid damage}

\subsection{Desulfurization of simple aminoacids}

After the discovery of the lipid isomerization and its feasibility under physiological conditions, it was important to address what can be the real culprits of such a process occurring in vivo. For this reason, a variety of biologically relevant sulfur-containing compounds were examined for their potentiality to produce thiyl radicals and induce lipid isomerisation, and sulfur-containing amino acid residues came into play. Reactive oxygen species (ROS), in particular ${ }^{\circ} \mathrm{OH}$ radicals, cause oxidative damage to several amino acids, including cysteine (Cys) and methionine (Met) (Bobrowski et al., 2007; Huang \& Rauk, 2004). Thiol moieties RSH can react under free radical stress (for example, caused by $\mathrm{HO}^{\circ}$ radicals) by donating hydrogen atoms, therefore giving thiyl radicals RS*. Actually, the hydrogen donation is still largely intended as a protective mechanism against radical degradation of cellular components, being the basic mechanism accepted for glutathione protective role from free radical degradation. Also some proteins, like albumin are thought to have such protective role in living systems [Roche et al., 2008]. It is worth underlining that, due to the high molecular weight, protein-bound thiyl radicals can have some restriction in the diffusion, therefore the ability to become an isomerising agent by reaching the double bond in the membrane bilayer should be considered in each case. Together with thiol, disulfide and thioether functions are also present in amino acid residues (cysteine, cystine and methionine) and are known to give a variety of reactions under free radical conditions (Alfassi, 1999). The interaction of reducing reactive species derived from the ionizing irradiation of water, namely hydrated electrons $\left(\mathrm{e}_{\mathrm{aq}}{ }^{-}\right)$and $\mathrm{H}^{\bullet}$ atoms, with disulfides in aqueous solution is well understood (Alfassi,1999). Figure 8 shows the disulfide radical anion (RSSR --) derived from the direct electron attachment and its equilibrium with the protonated form, the sulfuranyl radical, which is obtained also by the $\mathrm{H}^{\bullet}$ atom attack directly to the sulfide moiety. Both reactive species dissociate reversibly into two entities, RS ${ }^{\bullet}$ and RSH (or RS-). On the other hand, $\mathrm{H}^{\bullet}$ atoms were studied to selectively attack the thioether function of methionine, as shown in Figure 9 with the formation of a sulfuranyltype radical that gives a rapid desulfurization. From this intermediate a desulfurization is produced with formation of $\mathrm{CH}_{3} \mathrm{SH}$, a diffusible species that under radical stress condition exists as the corresponding thiyl radical $\mathrm{CH}_{3} \mathrm{~S}^{*}$. The desulfurization of methionine gives $\alpha$ aminobutyric acid (Aba), which corresponds to a chemical mutation to a naturallyoccurring, but not genetically-coded, amino acid.

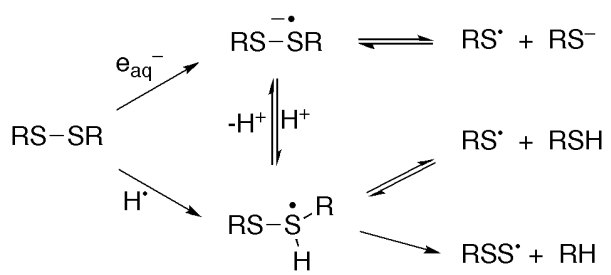

Fig. 8. The mechanism for reaction of $\mathrm{e}_{\mathrm{aq}}{ }^{-}$and $\mathrm{H}^{\bullet}$ atom attack with disulfide moiety in aqueous solution 


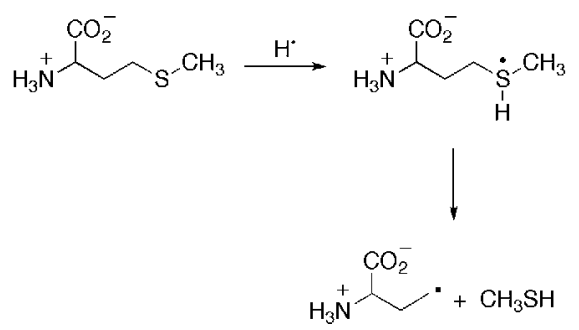

Fig. 9. The $\mathrm{H}^{\bullet}$ atom attack on the thioether moiety of methionine affords the sulfuranyl radical, which unimolecularly decomposes to give the carbon-centered radical and methanethiol.

The diffusible $\mathrm{CH}_{3} \mathrm{~S}^{\bullet}$ radicals are able to reach the lipid bilayer of a membrane model and cause double bond isomerisation with a very high efficiency, as studied with single amino acids and peptide sequences, like Met-enkephalin. Met-enkephalin (Tyr-Gly-Gly-Phe-Met) is known as an endogenous mediator interacting with opioid receptors, therefore with several biological activities in the field of pain control. The reactivity of this substrate under reductive conditions was to generate the corresponding desulfurated sequence, with Aba instead of Met residue, which is going to be studied for its biological effects compared to the natural sequence (Mozziconacci et al., 2007). Finally, the attack of $\mathrm{H}^{\bullet}$ atoms can occur also to the sulfur moiety of cysteine residues, leading again to desulfurization reaction with the formation of $\mathrm{H}_{2} \mathrm{~S}$ and the amino acid alanine (Ala), which represents a post-translational chemical modification of natural amino acids (Ferreri et al., 2008). It is worth noting that none of these transformations are yet related to radical stress in vivo, therefore future developments can be envisaged concerning the presence of desulfurized amino acid residues as markers of free radical damage.

\subsection{Radical-based modifications for protein libraries}

Biomimetic chemistry can be successfully applied to the discovery of free radical modifications of sulfur-containing substrates based on the formation of diffusible radical species. Indeed, the reversible addition of thiyl radicals to double bonds (see Figure 5) is interesting as is a specific radical process, and indirectly amplifies the effect of a few moles of thiyl radicals, thus allowing the damage to be envisaged even at low level. This can be useful in large structures such as proteins, where radicals can attack several reactive positions, and the individuation of damages can be somehow difficult. We developed the heterogeneous system of liposome vesicles added with protein substrates as a very sensitive and reliable tool for detecting radical damage at the level of S-containing residues. This first detection is then associated to proteomic analyses, and this approach is expected to open the way to molecular libraries useful for detecting damages in biological samples. We studied bovine pancreatic RNase A individuating both methionine and cystine reactive sites, with modification of Met and Cys to $\alpha$-amino butyric acid (Aba) and alanine (Ala) residues, respectively (Ferreri et al., 2006b; Ferreri et al., 2008). The sequence of amyloid $\beta$-peptide of 40 amino acids, where only the Met residue is present as a S-containing amino acid, was also studied for its connections with the alteration reported in the Alzheimer disease. In this case, reductive stress was able to produce the selective desulfurization of this sequence, and the identification of the resulting Aba-modified peptide has been possible (Kadlcik et al., 2006). 
More recently, metallothioneins (MTs), small proteins extremely rich in cysteines, have been analyzed. This high-Cys content, up to a $30 \%$ of their amino acids, confers them an exceptional capacity to coordinate heavy metals through the formation of metal-thiolate bonds. The presence of labile sulfide anions as non-proteic ligands was reported in the metal-MT complexes (Capdevila et al., 2005). Therefore, the presence of both sulfurcontaining amino acids and sulfide anions in the same molecular aggregate makes this system a very interesting case to determine the main targets of the reductive stress. Interestingly, a differentiation of reactivity can be assessed, since the reductive conditions seem to preferentially select the methionine and cysteine residues (Torreggiani et al., 2009). In all these experiments the protein damages were found to be coupled with the unsaturated lipid isomerisation and formation of trans fatty acids in the vesicles. As previously noted, liposomes containing double bonds are a very sensitive reporter system of the formation of diffusible thiyl radicals, since trans isomers are formed through the catalytic activity of Scentered radicals as isomerising agents. This system can be proposed as a first screening of the protein/peptide potential damage at the level of S-containing residues, in order to proceed with analytical identification of the exposed sites of radical attack along the sequence. This can be done by proteomic analysis using high resolution mass spectrometers, such as electrospray-quadrupole-time of flight (ESI-Q-TOF) instruments. Specific mass differences can be tentatively ascribed to a certain protein modification on the basis of the exact molecular mass of the atoms introduced/removed. Furthermore, a punctual characterization of the reductive modifications occurring on the protein primary structure is necessary for a better comprehension of the molecular damage produced; to this purpose, an in-deeper analysis at peptide/amino acid level has to be performed. As an example, the structural characterization of the peptide digest from irradiation-induced reductive modification of bovine pancreatic RNase A demonstrated that only 3 out of 8 Cys residues present within the whole protein primary structure were transformed into Ala, thus proving that two unique disulphide bonds were selectively reduced during reaction. These experiments also established that a unique Met residue, namely Met79, was reduced to Aba, while the remaining ones were oxidized with a variable degree, probably as consequence of the sample manipulation (Ferreri et al., 2008).

\section{Biological scenarios suggested by biomimetic models}

Biomimetic models can also be suggestive for scenarios that are not easily obtained directly from the biological reality, such as for example the scenario of formation of the first living cells, or protocell. This subject attracts interest from several points of view, either from the archeobiology, studying the creation of the first living entity from the primordial soup, and the biotechnology, looking for a nanosized bioreactor where biosynthesis can be created upon needs. In the former case, unilamellar liposomes are used as model for the "minimal cell", as form of minimal life that is, for assessing the minimal and sufficient conditions allowing for the start of cellular evolution. This can be viewed also as the minimal genome needed for creating the enzymatic functioning and replication of the information, but several insights can also be obtained from the point of view of the minimal physical dimension of the cell to permit the life. This section cannot comment on the various aspects related to the protocell experiments, that are treated in several reviews and books. However, it is important to focus on an aspect that emerges also from the study of the above reported 
lipid cis-trans isomerization, that concerns the type of lipids used for this minimal cell experiments. Looking at the membrane composition of the first forms of life, most of the fatty acids were saturated and branched hydrocarbon chains, that defines a very small compartment of a few hundreds of nanometers. To obtain larger compartments such as those of prokaryotes ranging from 300 to 1000 nanometers and those of eukaryotes up to 2500 nanometers and more, lipids had to be differentiated (Seckbach, 2004). In this vision, the large variety offered by the families of saturated, monounsaturated and polyunsaturated fatty acids can be rationalized on the basis of their contribution to cell dimension. To deepen this subject, liposomes prepared by the injection methodology can be used because they well represent the spontaneous generation of vesicles. The resulting diameter of the cell compartment should be directly related to the type of the lipids used in the starting mixture. In this respect, a preliminary question must be addressed on the role of lipid geometry since the naturally occurring geometry in all eukaryotic cells is the cis arrangement, with exclusion of the trans isomers, which are only present in the prokaryote world where they instead mean survival (Heipieper et al., 2003). However, if the primordial conditions are considered, it is likely that, under high temperatures and irradiation, the lipid geometry presented both isomeric configurations, therefore it cannot be excluded that the cell compartment contained both forms. To verify such possibility and the corresponding compartmental dimension, different phospholipid structures were used to form unilamellar liposomes, including saturated, monounsaturated cis and trans lipids. In Table 1 the dimension obtained from dipalmitoylphosphatidylcholine (with saturated fatty acid residues of palmitic acid, 16:0) is compared with the liposomes obtained with 1-palmitoyl-2oleoylphosphatidylcholine (with one chain of saturated fatty acid residues of palmitic acid, 16:0 and the other chain of the monounsaturated cis fatty acid, oleic acid 9cis-18:1) and those obtained with 1-palmitoyl-2-elaidoylphosphatidylcholine (with one chain of saturated fatty acid residues of palmitic acid, 16:0 and the other chain of the monounsaturated trans fatty acid, oleic acid 9trans-18:1) (Ferreri et al., 2006a).

\begin{tabular}{|l|l|}
\hline Phospholipid & Diameter $(\mathrm{nm})$ \\
\hline POPC & $97 \pm 10$ \\
\hline PEPC 83\% & $72 \pm 8$ \\
\hline DPPC & $45 \pm 8$ \\
\hline
\end{tabular}

Table 1. Dimension of liposomes obtained by the injection methodology from different phospholipids

It is clearly seen that saturated fatty acids are the smallest compartment followed by trans fatty acids, whereas the cis configuration is ensuring the widest compartment possible. Also the properties of the bilayer obtained by the different fatty acids showed consistent differences, as expected by the different physical parameters of the lipids (Ferreri et al., 2006c). Recent results confirmed the dimension theory since protein synthesis could be obtained in $100 \mathrm{~nm}$-vesicles, formed by POPC where an ensemble made of enzymes, ribosomes, tRNA and low molecular weight molecules was enclosed (Pereira de Souza et al., 2009). This indicated the minimal cell dimension where a biosynthetic machinery can function. Extension of these results can lead to a thorough study of all possible combinations of lipid structures, which are compatible with the dimension and the functioning of the minimal cell. Indeed, the most important contribution to the dimension of the compartment 
delimited by membranes is given by the cis-unsaturated geometry, therefore the variety of the polyunsaturated fatty acids present in the biological membranes can find its original reason in the creation of the best compartment compatible with life. From the studies carried out so far in liposomes, it can be logically inferred that, once formed, a minimal cell entity which certainly included cis and trans lipids, underwent a natural selection based on the resulting biophysical and biosynthetic capabilities, which above all excluded the trans geometry from eukaryotic membranes.

\section{Conclusions}

The chapter offered an overview of main processes studied by the membrane model of liposomes. The use of liposomes as carrier for drugs and active compound delivery cannot be forgotten, and some aspects of the liposome technology and reactivity shown in this chapter can have relevance also in this field. The system of monolamellar liposomes is expected to be extensively used for the examination of free radical reactivity, especially from a chemical biology approach exploring the biomimetic chemistry of radical species in detail, where the experimental set up of the liposomal model can satisfactorily represent the biological scenarios. In this context an improvement of interdisciplinarity is needed, among the fields of chemistry, biochemistry, biology and medicine, in order to create a common territory where the achievements of free radical reactivity can be straightforwardly transferred to a better comprehension of the biological pathways in health and diseases.

\section{References}

Alfassi, Z. B. , (Ed.) (1999). S-centered radicals, Wiley, Chichester

Barber, D.J.W. \& Thomas, J.K. (1978). Reactions of radicals with lecithin bilayers. Radiat. Res. 74, 51-65

Barclay, L.R.C. \& Ingold, K.U. (1981). Autoxidation of biological molecules. 2. The autoxidation of a model membrane. A comparison of the autoxidation of egg lecithin phosphatidylcholine in water and in chlorobenzene. J. Am. Chem. Soc. 103, 6478-6485

Barclay, L.R.C.; Locker, S.J.; MacNeil, J.M. \& Vankessel, J. (1985). Quantitative studies of the autoxidation of linoleate monomers sequestered in phosphatidylcholine bilayers. Absolute rate constants in bilayer. Can. J. Chem. 63, 2633-2638.

Barclay, L.R.C. (1993). 1992 Syntex Award Lecture. Model biomembranes: quantitative studies of peroxidation, antioxidant action, partitioning, and oxidative stress. Can. J. Chem. 71, 1-16

Bobrowski, K.; Hug, G.L.; Pogocki, D.; Marciniak, B. \& Schoeneich, C. (2007). Stabilization of Sulfide Radical Cations through Complexation with the Peptide Bond: Mechanisms Relevant to Oxidation of Proteins Containing Multiple Methionine Residues. J. Phys. Chem. B 111, 9608-9620

Buettner, G.R. (1993). The pecking order of free radicals and antioxidants: lipid peroxidation, alpha-tocopherol, and ascorbate. Arch. Biochem. Biophys. 300, 535-43

Capdevila, M.; Domènech, J.; Pagani, A.; Tío, L.; Villarreal, L. \& Atrian, S. (2005). Zn- and Cd-Metallothionein Recombinant Species from the Most Diverse Phyla May Contain Sulfide (S2-) Ligands. Angew. Chem. Int. Ed. 44, 4618-4622 
Chatgilialoglu, C.; Zambonin, L.; Altieri, A.; Ferreri, C.; Mulazzani, Q.G. \& Landi, L. (2002). Geometrical Isomerism of Monounsaturated Fatty Acids. Thiyl Radical Catalysis and Influence of Antioxidant Vitamins. Free Radic. Biol. Med. 33,1681-1692

Chatgilialoglu, C. \& Ferreri, C. (2005). Trans Lipids: The Free Radical Path. Acc. Chem. Res. 36, $441-448$

Chatgilialoglu, C.; Ferreri, C.; Lykakis, I.N. \& Wardman, P. (2006). Trans fatty acids and radical stress: what are the real culprits? Bioorg. Med. Chem. 14, 6144-6148

Cutler, R.G. \& Rodriguez, H., (Eds.), (2003). Critical Reviews of oxidative stress and aging, World Scientific, New Yersey

Domazou, A.S. \& Luisi, P.L. (2002). Size distribution of spontaneously formed liposomes by the alcohol injection method. J. Liposome Res. 12, 205-220

Esterbauer, H.; Striegl, G.; Puhl, H. \& Rotheneder, M. (1989). Continuous monitoring of in vitro oxidation of human low density lipoprotein. Free Radic. Res. Commun. 6, 67-75

Fendler, J.H. \& Romero, A. (1977). Liposomes as drug carriers. Life Sci. 20, 1109-1120

Ferreri, C.; Costantino, C.; Landi, L.; Mulazzani, Q.G. \& Chatgilialoglu C. (1999). The Thiyl radical Mediated Isomerization of cis-Monounsaturated Fatty Acid Residues in Phospholipids: A Novel path of membrane Damage? Chem. Commun. 407-408

Ferreri, C.; Costantino, C.; Perrotta, L.; Landi, L.; Mulazzani, Q.G. \& Chatgilialoglu, C. (2001). Cis-trans isomerization of polyunsaturated fatty acid residues in phospholipids catalysed by thiyl radicals. J. Am. Chem. Soc. 123, 4459-4468

Ferreri, C.; Faraone-Mennella, M.R.; Formisano, C.; Landi, L. \& Chatgilialoglu, C. (2002). Arachidonate geometrical isomers generated by thiyl radicals: the relationship with trans-lipids detected in biological samples. Free Radic. Biol. Med. 33, 1516-1526

Ferreri, C.; Samadi, A.; Sassatelli, F.; Landi, L. \& Chatgilialoglu, C. (2004a). Regioselective cis-trans-isomerization of arachidonic double bonds by thiyl radicals: the influence of phospholipids supramolecular organization. J. Am. Chem. Soc. 126, 1063-1072

Ferreri, C.; Kratzsch, S.; Brede, O.; Marciniak, B. \& Chatgilialoglu, C. (2004b). Trans lipid formation induced by thiols in human monocytic leukemia cells. Free Radic. Biol. Med. 38, 1180-1187

Ferreri, C.; Angelini, F.; Chatgilialoglu, C.; Dellonte, S.; Moschese, V.; Rossi, P. \& Chini L. (2005a). Trans fatty acids and atopic eczema/dermatitis syndrome: the relationship with a free radical cis.trans isomerization of membrane lipids. Lipids 40, 661-667

Ferreri, C.; Kratzsch, S.; Landi, L. \& Brede, O. (2005b). Thiyl radicals in biosystems: effects on lipid structures and metabolism. Cell. Mol. Life Sci. 62, 834-847

Ferreri, C. \& Chatgilialoglu, C. (2005c). Geometrical trans lipid isomers: a new target for lipidomics. ChemBioChem 6, 1722-1734

Ferreri, C.; Pierotti, S.; Barbieri, A.; Zambonin, L.; Landi, L.; Rasi, S.; Luisi, P.L.; Barigelletti, F. \& Chatgilialoglu, C. (2006a). Comparison of phosphatidylcholine vesicle properties related to geometrical isomerism. Photochem. Photobiol. 82, 274-280

Ferreri, C.; Manco, I.; Faraone-Mennella, M.R.; Torreggiani, A.; Tamba, M.; Manara, S. \& Chatgilialoglu, C. (2006b). The reaction of hydrogen atoms with methionine residues: a model of reductive radical stress causing tandem protein-lipid damage. ChemBioChem 7, 1738-1744

Ferreri, C.; Pierotti, S.; Chatgilialoglu, C.; Barbieri A. \& Barigelletti, F. (2006c) Probing the influence of cis-trans isomers on model lipid membrane fluidity using cis-parinaric acid and stop-flow technique. Chem. Commun., 529-531 
Ferreri, C.; Chatgilialoglu, C.; Torreggiani, A.; Salzano, A. M.; Renzone, G. \& Scaloni, A. (2008). The reductive desulfurization of Met and Cys residues in bovine RNase A is associated with trans lipids formation in a mimetic model of biological membranes. J. Proteome Res. 7, 2007-2015

Fox, B.G.; Lyle, K.S. \& Rogge, C.E. (2004). Reactions of the diiron enzyme stearoyl-acyl carrier protein desaturase. Acc. Chem. Res. 37, 421-429

Goldberg, I.H. (1987). Free radical mechanisms in neocarzinostatin-induced DNA damage. Free Radic. Biol. Med. 3, 41-54

Gregoriadis, G. (1992). Liposome Technology, 2nd Edition, Vol 2, CRC Press, Boca Raton

Halliwell, B. \& Gutteridge, J.M.C. (Eds.) (2000). Free Radicals in Biology and Medicine, 3rd ed.; Clarendon Press, Oxford, U.K

Heipieper, H.J.; Meinhardt, F. \& Segura A. (2003) The cis-trans isomerase of unsaturated fatty acids in Pseudomonas and Vibrio: biochemistry, molecular biology and physiological function of a unique stress adaptive mechanism. FEMS Microbiol. Lett. 229, 1-7

Huang. M.L. \& Rauk, A. (2004). Reactions of One-Electron-Oxidized Methionine with Oxygen: An ab Initio Study. J. Phys. Chem. A 108, 6222-6230

Jiang, H.; Kruger, N.; Lahiri, D.R.; Wang, D.; Vatèle, J.-M. \& Balazy, M. (1999). Nitrogen dioxide induces cis-trans isomerization of arachidonic acid within cellular phospholipids. J. Biol. Chem. 274, 16235-16241

Kadiiskaa, M.B.; Gladena, B.C.; Bairda, D.D.; Germoleca, D.; Grahama, L.B. et al. (2005). Biomarkers of Oxidative Stress Study II. Are oxidation products of lipids, proteins, and DNA markers of $\mathrm{CCl}_{4}$ poisoning? Free Radic. Biol. .Med. 38, 698-710

Kadlcik, V.; Sicard-Roselli, C.; Houée-Levin, C.; Ferreri, C. \& Chatgilialoglu, C. (2006). Reductive modification of methionine residue in amyloid $\beta$-peptide. Angew. Chem. Int. Ed. 45, 2595-2598

Konings, A.W.T.; Damen, J. \& Trieling, W.B. (1979). Protection of liposomal lipids against radiation induced oxidative damage. Int. J. Radiat. Biol. 35, 343-350

Lasic, D.D. (1993). Liposomes: from physics to applications. Elsevier, Amsterdam

MacDonald, R.C.; MacDonald, R.I.; Menco, B.P.; Takeshita, K.; Subbarao, N.K. \& Hu, L.R. (1991). Small-volume extrusion apparatus for preparation of large, unilamellar vesicles. Biochim. Biophys. Acta 1061, 297-303

Marathe, D. \& Mishra, K.P. (2002). Radiation -induced changes in permeability in unilamellar phospholipids liposomes. Radiation Res. 157, 685-692

Marnett, L.J. (2000). Cyclooxygenase mechanisms. Curr. Opin. Chem. Biol., 4, 545-552

Mihaljević, B.; Katušin-Ražem, B. \& Ražem, D. (1996). The reevaluation of the ferric thiocyanate assay for lipid hydroperoxides with special considerations of the mechanistic aspects of the response. Free Radic. Biol. Med. 21, 53-63

Milne, G.L. \& Porter, N.A. (2001). Separation and identification of phospholipid peroxidation products. Lipids 36, 1265-1275

Mishra, K.P. (2004). Cell membrane oxidative damage induced by gamma-radiation and apoptotic sensitivity. J. Environ. Path Toxicol. Oncol. 23, 61-66

Mozziconacci, O.; Bobrowski, K.; Ferreri, C. \& Chatgilialoglu, C. (2007). Reaction of hydrogen atom with Met-enkephalin and related peptides. Chem. Eur. J. 13, 20292033 
Nakazawa, T. \& Nagatsuka, S. (1980). Radiation-induced lipid peroxidation and membrane permeability in liposomes. Int. J. Radiat. Biol. 38, 537-544

New, R.R.C. (1990). Liposomes: a practical approach. IRL Press, Oxford

Niki, E.; Kawakami, A.; Yamamoto, Y. \& Kamiya, Y. (1985). Oxidation of lipids. VIII. Synergistic inhibition of oxidation of phosphatidylcholine liposome in aqueous dispersion by vitamin E and vitamin C. Bull. Chem. Soc. Jpn. 58, 1971-1975

Pereira de Souza, T.; Stano, P. \& Luisi, P.L. (2009) The minimal size of liposome-based model cells brings about a remarkably enhanced entrapment and protein synthesis. ChemBioChem 10, 1056-1063.

Porter, N.A.; Wolf, R.A.; Yarbro, E.M. \& Weenen, H. (1979). The autoxidation of arachidonic acid: formation of the proposed SRS-A intermediate. Biochem. Biophys. Res. Commun. 89, 1058-1064

Porter, N.A.; Weber, B.A.; Weenen, H. \& Khan, J.A. (1980). Autoxidation of polyunsaturated lipids. Factors controlling the stereochemistry of product peroxides. J. Am. Chem. Soc. 102, 5597-5601

Prager, A., Terry, N.H.A. \& Murray, D. (1993). Influence of intracellular thiol and polyamine levels on radioprotection by aminothiols. Int. J. Radiat. Biol. 64, 71-81

Pryor, W.A. \& Godber, S.S. (1991). Noninvasive measures of oxidative stress status in humans. Free Radic. Biol. Med. 10, 177-184

Puca, A.A.; Novelli, V.; Viviani, C.; Andrew, P.; Somalvico, F.; Cirillo, N.A.; Chatgilialoglu, C. \& Ferreri, C. (2008). Lipid profile of erythrocyte membranes as possible biomarker of longevity. Rejuven. Res. 11, 63-72

Reichard, P. \& Ehrenberg, A. (1983). Ribonucleotide reductase: a radical enzyme. Science 221, 514-519

Roche, M.; Rondeau, P.; Singh, N.R.; Tarnus, E. \& Bourdon, E. (2008). The antioxidant properties of serum albumin. FEBS Lett. 582, 1783-1787

Sébédio, J.-L. \& Christie, W.W. (Eds.) (1998). Trans Fatty Acids in Human Nutrition. The Oily Press, Dundee

Seckbach, J. (Ed.) (2004). Origins. Genesis, evolution and diversity of life. Kluwer, Dordrecht

Schnitzer, E.; Pinchuk, I. \& Lichtenberg, D. (2007). Peroxidation of liposomal lipids. Eur. Biophys. J. 36, 499-515

Siminovitch, D.J.; Wong, P.T.T. \& Mantsch, H.H. (1987). Effects of cis and trans unsaturation on the structure of phospholipids bilayers: a high-pressure infrared spectroscopic study. Biochemistry 26, 3277-3278

Torreggiani, A.; Domènech. J.; Orihuela, R.; Ferreri, C.; Atrian, S.; Capdevila, M. \& Chatgilialoglu, C. (2009). Zinc and Cadmium Complexes of a Plant Metallothionein under Radical Stress: Desulfurisation Reactions Associated with the Formation of trans-Lipids in Model Membranes. Chem. Eur. J. 15, 6015-6024

Vance, D.E. \& Vance, J.E., (Eds), (2002). Biochemistry of lipids, lipoproteins and membranes, 4th ed., Elsevier, Amsterdam

Wolff, R.L. \& Entressangles, B. (1994). Steady-state fluorescence polarization study of structurally defined phospholipids from liver mitochondria of rats fed elaidic acid. Biochim. Biophys. Acta 1211, 198-206

Zambonin, L.; Ferreri, C.; Cabrini, L.; Prata, C.; Chatgilialoglu, C. \& Landi, L. (2006). Occurrence of trans fatty acids in rats fed a trans-free diet: a free radical-mediated formation? Free Radic. Biol. Med. 40, 1549-1556 


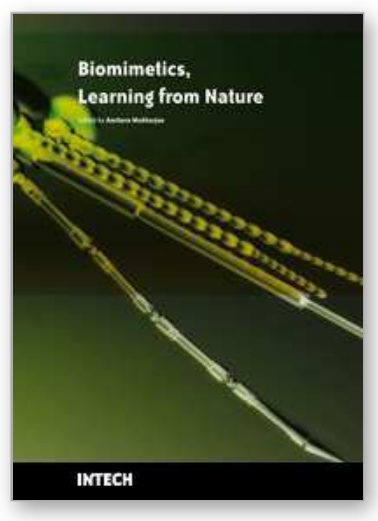

\section{Biomimetics Learning from Nature}

Edited by Amitava Mukherjee

ISBN 978-953-307-025-4

Hard cover, 534 pages

Publisher InTech

Published online 01, March, 2010

Published in print edition March, 2010

Nature's evolution has led to the introduction of highly efficient biological mechanisms. Imitating these mechanisms offers an enormous potential for the improvement of our day to day life. Ideally, by bio-inspiration we can get a better view of nature's capability while studying its models and adapting it for our benefit. This book takes us into the interesting world of biomimetics and describes various arenas where the technology is applied. The 25 chapters covered in this book disclose recent advances and new ideas in promoting the mechanism and applications of biomimetics.

\section{How to reference}

In order to correctly reference this scholarly work, feel free to copy and paste the following:

Chryssostomos Chatgilialoglu and Carla Ferreri (2010). Biomimetic Chemistry: Radical Reactions in Vesicle Suspensions, Biomimetics Learning from Nature, Amitava Mukherjee (Ed.), ISBN: 978-953-307-025-4, InTech, Available from: http://www.intechopen.com/books/biomimetics-learning-from-nature/biomimetic-chemistryradical-reactions-in-vesicle-suspensions

\section{INTECH}

open science | open minds

\section{InTech Europe}

University Campus STeP Ri

Slavka Krautzeka 83/A

51000 Rijeka, Croatia

Phone: +385 (51) 770447

Fax: +385 (51) 686166

www.intechopen.com

\section{InTech China}

Unit 405, Office Block, Hotel Equatorial Shanghai

No.65, Yan An Road (West), Shanghai, 200040, China

中国上海市延安西路65号上海国际贵都大饭店办公楼 405 单元

Phone: +86-21-62489820

Fax: +86-21-62489821 
(C) 2010 The Author(s). Licensee IntechOpen. This chapter is distributed under the terms of the Creative Commons Attribution-NonCommercialShareAlike-3.0 License, which permits use, distribution and reproduction for non-commercial purposes, provided the original is properly cited and derivative works building on this content are distributed under the same license. 